\title{
Refined Hardy inequalities
}

\author{
Hajer Bahouri, JeAn-Yves Chemin And Is ABelle Gallagher
}

\begin{abstract}
The aim of this article is to present "refined" Hardy-type inequalities. Those inequalities are generalisations of the usual Hardy inequalities, their additional feature being that they are invariant under oscillations: when applied to highly oscillatory functions, both sides of the refined inequality are of the same order of magnitude. The proof relies on paradifferential calculus and Besov spaces. It is also adapted to the case of the Heisenberg group.
\end{abstract}

Mathematics Subject Classification (2000): 43A80 (primary); 42B99 (secondary).

\section{Introduction}

The aim of this article is to prove a "refined" version of the Hardy inequalities [11, 12]. Those inequalities have some importance in Analysis (among other applications we can mention blow-up methods or the study of pseudodifferential operators with singular coefficients). Many works have been devoted to those inequalities, and our goal is first to provide an elementary proof of the standard Hardy inequality, and then to prove a refined inequality in the spirit of the refined Sobolev inequality proved in [10]. The setting will be both the classical $\mathbf{R}^{N}$ space, as well as the Heisenberg group $\mathbf{H}^{d}$ (for an application of the Hardy inequality on the Heisenberg group we refer for instance to [1]).

\subsection{Elementary Hardy inequality}

The simple case of $\mathbf{R}^{N}$ with $N \geq 3$ with one derivative gives the following inequality:

$$
\int_{\mathbf{R}^{N}} \frac{u^{2}(x)}{|x|^{2}} d x \leq C\|\nabla u\|_{L^{2}}^{2} .
$$

In order to prove this inequality, it is enough to observe that we have

$$
\frac{1}{|x|^{2}}=-\frac{1}{2} R\left(\frac{1}{|x|^{2}}\right) \quad \text { with } \quad R=x \cdot \nabla \text {. }
$$

Received February 22, 2006; accepted in revised form July 21, 2006. 
An integration by parts joint with the fact that the divergence of $R$ is equal to $N$ gives the result.

Let us now present the case of the Heisenberg group. The Heisenberg group $\mathbf{H}^{d}$ is the space $\mathbf{R}^{2 d+1}$ endowed with the following product group law:

$$
w \cdot w^{\prime}=\left(x+x^{\prime}, y+y^{\prime}, s+s^{\prime}+\left(y \mid x^{\prime}\right)-\left(y^{\prime} \mid x\right)\right)
$$

where $w=(x, y, s)$ and $w^{\prime}=\left(x^{\prime}, y^{\prime}, s^{\prime}\right)$. Let us notice that $\mathbf{H}^{d}$ is a non commutative group and that the inverse of $w$ is $w^{-1}=(-x,-y,-s)$. The Lebesgue measure on $\mathbf{H}^{d}$ seen as $\mathbf{R}^{2 d+1}$ is invariant by translation with respect to this law. We define the convolution of two functions by

$$
f \star g\left(w^{\prime}\right)=\int_{\mathbf{H}^{d}} f\left(w^{\prime} w^{-1}\right) g(w) d w .
$$

Let us emphasize that this convolution product is, as $\mathbf{H}^{d}$ itself, not commutative. We say that a vector field $X$ is left invariant if $X(f(a \cdot))=(X f)(a \cdot)$. The Lie algebra of left invariant vector fields is spanned by the vector fields

$X_{j}=\partial_{x_{j}}+y_{j} \partial_{s}, Y_{j}=\partial_{y_{j}}-x_{j} \partial_{s} \quad$ with $j \in\{1, \ldots, d\} \quad$ and $\quad S=\partial_{s}=\frac{1}{2}\left[Y_{j}, X_{j}\right]$.

In all that follows, we shall denote by $\mathcal{Z}$ the family defined by $Z_{j}=X_{j}$ and $Z_{j+d}=$ $Y_{j}$. Let us denote

$$
\Delta_{\mathbf{H}} \stackrel{\text { def }}{=} \sum_{j=1}^{2 d} Z_{j}^{2} \quad \text { and for } \alpha \in\{1, \ldots, 2 d\}^{k}, \quad \mathcal{Z}^{\alpha} \stackrel{\text { def }}{=} Z_{\alpha_{1}} \ldots Z_{\alpha_{k}} .
$$

One can associate Sobolev spaces to the system $\mathcal{Z}$ through the following definition.

Definition 1.1. Let $k$ be a non negative integer, we denote by $\dot{H}^{k}\left(\mathbf{H}^{d}\right)$ the homogeneous Sobolev space of order $k$ which is the space of functions $u$ such that

$$
\|u\|_{\dot{H}^{k}\left(\mathbf{H}^{d}\right)}^{2} \stackrel{\text { def }}{=} \sum_{\alpha \in\{1, \ldots, 2 d\}^{k}}\left\|\mathcal{Z}^{\alpha} u\right\|_{L^{2}\left(\mathbf{H}^{d}\right)}^{2}<\infty .
$$

Let us also introduce the distance to the origin

$$
\rho(w) \stackrel{\text { def }}{=}\left(\left(|x|^{2}+|y|^{2}\right)^{2}+s^{2}\right)^{\frac{1}{4}} \quad \text { with } \quad w=(x, y, s)
$$

and the dilation $\delta_{\lambda}(w) \stackrel{\text { def }}{=}\left(\lambda x, \lambda y, \lambda^{2} s\right)$. Let us point out that the function $\rho$ is homogenenous of degree 1 in the sense that

$$
\rho \circ \delta_{\lambda}=\lambda \rho
$$


and the vector fields $Z_{j}$ change the homogeneity as

$$
Z_{j}\left(f \circ \delta_{\lambda}\right)=\lambda\left(Z_{j} f\right) \circ \delta_{\lambda}
$$

Moreover, we have

$$
\left|Z_{j} \rho^{\sigma}\right| \leq C_{\sigma} \rho^{\sigma-1}
$$

Let us also introduce the homogeneous dimension $N=2 d+2$ noticing that the Jacobian of the dilation $\delta_{\lambda}$ is $\lambda^{N}$. The Hardy inequality with one derivative in this context is

$$
\int_{\mathbf{H}^{d}} \frac{u^{2}(w)}{\rho^{2}(w)} d w \leq C\left\|\nabla_{\mathbf{H}} u\right\|_{L^{2}}^{2} \quad \text { where } \quad \nabla_{\mathbf{H}} u \stackrel{\text { def }}{=}\left(Z_{1} u, \ldots, Z_{2 d} u\right) .
$$

The proof (as written for instance in [1]) of this inequality relies mainly on the fact that

$$
\frac{1}{\rho^{2}}=-\frac{1}{2} R\left(\frac{1}{\rho^{2}}\right) \quad \text { with } \quad R \stackrel{\text { def }}{=} \sum_{j=1}^{d}\left(x_{j} X_{j}+y_{j} Y_{j}\right)+2 s \partial_{s} .
$$

An integration by parts and the fact that $\operatorname{div} R=N$ essentially gives the result.

\subsection{More general Hardy inequalities}

Now we want to state Hardy inequalities with any number of derivatives less than $N / 2$.

Theorem 1.2. Let $s \in] 0, N / 2[$. There exists a constant $C$ such that

$$
\int_{\mathbf{R}^{N}} \frac{|u|^{2}(x)}{|x|^{2 s}} d x \leq C\|u\|_{\dot{H}^{s}\left(\mathbf{R}^{N}\right)}^{2} \quad \text { and } \quad \int_{\mathbf{H}^{d}} \frac{|u|^{2}(w)}{\rho^{2 s}(w)} d w \leq C\|u\|_{\dot{H}^{s}\left(\mathbf{H}^{d}\right)}^{2}
$$

where the spaces $\dot{H}^{s}$ are defined by complex interpolation.

Classically, the way of proving this consists in proving that the operators

$$
\frac{1}{|x|^{s}}(-\Delta)^{-\frac{s}{2}} \text { or } \frac{1}{\rho^{s}}\left(-\Delta_{\mathbf{H}}\right)^{-\frac{s}{2}}
$$

are bounded on $L^{2}\left(\mathbf{R}^{N}\right)$ or $L^{2}\left(\mathbf{H}^{d}\right)$. The purpose of this paper is first to give a more direct proof of these inequalities, which will be the same for $\mathbf{R}^{N}$ or $\mathbf{H}^{d}$. Moreover, in the case of $\mathbf{R}^{N}$, let us apply the above Hardy inequality with $s=1$ to the family $\left(f_{\varepsilon}\right)_{\varepsilon>0}$ of functions defined by

$$
f_{\varepsilon}(x)=e^{i \frac{x_{1}}{\varepsilon}} \theta(x)
$$

where $\theta$ is a given function in the Schwartz class $\mathcal{S}\left(\mathbf{R}^{N}\right)$. The left-hand side of the inequality is obviously independent of $\varepsilon$ and the right-hand side is of order $\varepsilon^{-1}$. 
The second purpose of this paper is to improve Hardy inequalities into inequalities which in particular will be invariant under the multiplication by oscillating functions like $e^{i \frac{(x \mid \omega)}{\varepsilon}}$.

This requires the introduction of Besov spaces of negative index and thus Littlewood Paley theory. In the case of $\mathbf{R}^{N}$, this is quite classical. In the case of the Heisenberg group, it was constructed by H. Bahouri, P. Gérard and C.-J. Xu in [2] (see also [3]). We can summarize this theory in the following properties, which hold regardless of the space which can be $\mathbf{R}^{N}$ or $\mathbf{H}^{d}$; one of the features of this paper is to write unified statements and proofs, which hold independently of the space. It is therefore natural to introduce unified notation. In the same way as on the Heisenberg group we have defined a family $\mathcal{Z}$ of vector fields, we will denote on $\mathbf{R}^{N}$

$$
\text { for } \alpha \in\{1, \ldots, N\}^{k}, \quad \mathcal{Z}^{\alpha} \stackrel{\text { def }}{=} X_{\alpha_{1}} \ldots X_{\alpha_{k}}, \quad \text { where } \quad X_{\alpha_{j}} \stackrel{\text { def }}{=} \partial_{x_{\alpha_{j}}} \text {. }
$$

We will also use the following notation:

$$
\forall w \in \mathbf{R}^{N}, \quad w^{-1}=-w, \quad \rho(w) \stackrel{\text { def }}{=}\left(\sum_{j=1}^{N}\left|w_{j}\right|^{2}\right)^{\frac{1}{2}}, \quad \text { and } \quad \forall a \in \mathbf{R}, \delta_{a} w=a w .
$$

Using that notation, the elements of Littlewood-Paley theory we will need are the following.

Both in the case of $\mathbf{R}^{N}$ and $\mathbf{H}^{d}$, there exists a family $\left(S_{j}\right)_{j \in \mathbf{Z}}$ of operators such that for any $p$ belonging to $[1, \infty[$,

$$
\forall u \in L^{p}, \lim _{j \rightarrow-\infty}\left\|S_{j} u\right\|_{L^{p}}=0 \quad \text { and } \quad \lim _{j \rightarrow \infty}\left\|S_{j} u-u\right\|_{L^{p}}=0 .
$$

Moreover, for any multi-index $\alpha$, there exists a constant $C$ such that, for any $(p, q) \in$ $[1, \infty]^{2}$ satisfying $p \leq q$, we have

$$
\left\|\mathcal{Z}^{\alpha} S_{j} u\right\|_{L^{q}} \leq C 2^{j N\left(\frac{1}{p}-\frac{1}{q}\right)+|\alpha| j}\left\|S_{j} u\right\|_{L^{p}}
$$

Moreover, if $\Delta_{j} \stackrel{\text { def }}{=} S_{j+1}-S_{j}$, two integers $N_{0}$ and $N_{1}$ exist such that

$$
\begin{array}{r}
\left|j-j^{\prime}\right| \geq N_{0} \Longrightarrow\left(\Delta_{j} \Delta_{j^{\prime}}=0 \quad \text { and } \Delta_{j}\left(S_{j^{\prime}-N_{0}} u \Delta_{j^{\prime}} v\right)=0\right) \\
\left(\left|k-k^{\prime}\right| \leq N_{0} \quad \text { and } \quad j \geq k+N_{1}\right) \Longrightarrow \Delta_{j}\left(\Delta_{k} u \Delta_{k^{\prime}} v\right)=0 .
\end{array}
$$

For any positive integer $k$, there exists a constant $C$ such that, for any $p \in[1, \infty]$,

$$
\left\|\Delta_{j} u\right\|_{L^{p}} \leq C 2^{-2 j k}\left\|(-\Delta)^{k} \Delta_{j} u\right\|_{L^{p}} .
$$


The operators $\Delta_{j}$ are of the form

$$
\Delta_{j} u=u \star h_{j} \quad \text { with } h_{j}(w)=2^{j N} h\left(\delta_{2^{j}} w\right) \quad \text { and } h \in \mathcal{S} .
$$

We remark that as $\Delta_{j}$ is a function of the Laplacian (respectively sublaplacian) on $\mathbf{R}^{N}$ (respectively $\mathbf{H}^{d}$ ), it commutes with the latter operator.

Definition 1.3. Let $s \in \mathbf{R}$ be given, as well as $p$ and $r$, two real numbers in the interval $[1, \infty]$. Then we define the space $\dot{B}_{p, r}^{s}$ of tempered distributions $u$ such that

$$
\lim _{j \rightarrow-\infty} S_{j} u=0 \quad \text { and } \quad\|u\|_{\dot{B}_{p, r}^{s}} \stackrel{\text { def }}{=}\left\|\left(2^{j s}\left\|\Delta_{j} u\right\|_{L^{p}}\right)\right\|_{\ell^{r}(\mathbf{Z})}<\infty .
$$

Let us notice that Inequality (1.6) implies immediately that, when $q \geq p$ and $r^{\prime} \geq r$, we have

$$
\|u\|_{\dot{B}_{q, r^{\prime}}^{s-N}\left(\frac{1}{p}-\frac{1}{q}\right)} \leq C\|u\|_{\dot{B}_{p, r}^{s}} .
$$

The result we will prove is the following. It is stated and proved indifferently in $\mathbf{R}^{N}$ and $\mathbf{H}^{d}$.

Theorem 1.4. Let $s$ be a real number in the interval $] 0, N / 2[$ and let $p$ and $q$ be two real numbers in $[1, \infty]$ such that

$$
2 \leq q<\frac{2 N}{N-2 s}<p \leq \infty .
$$

There is a constant $C$ such that, for any function $u \in \dot{B}_{q, 2}^{s-N\left(\frac{1}{2}-\frac{1}{q}\right)}$, the following inequality holds:

$$
\begin{aligned}
\left(\int \frac{|u(w)|^{2}}{\rho^{2 s}(w)} d w\right)^{\frac{1}{2}} \leq C\|u\|_{\dot{B}_{p, 2}^{s-N\left(\frac{1}{2}-\frac{1}{p}\right)}}^{\alpha}\|u\|_{\dot{B}_{q, 2}^{s-N\left(\frac{1}{2}-\frac{1}{q}\right)}}^{1-\alpha} \\
\text { with } \quad \alpha=\frac{p q}{p-q}\left(\frac{1}{q}-\frac{1}{2}+\frac{s}{N}\right) .
\end{aligned}
$$

Let us remark that, when $p=\infty$ and $q=2$, the above theorem implies that

$$
\left(\int \frac{|u(w)|^{2}}{\rho^{2 s}(w)} d w\right)^{\frac{1}{2}} \leq C\|u\|_{\dot{B}_{\infty, 2}^{s-\frac{N}{2}}}^{\frac{2 s}{N}}\|u\|_{\dot{H}^{s}}^{1-\frac{2 s}{N}} .
$$

This inequality should be compared to the following similar result derived by $\mathrm{P}$. Gérard, Y. Meyer and F. Oru in [10], in the case of the Sobolev inequalities on $\mathbf{R}^{N}$ (see [3] for the Heisenberg case), namely

$$
\|u\|_{L^{r}} \leq C\|u\|_{\substack{\dot{B}_{\infty, \infty}^{s-\frac{N}{2}} \\ \frac{2 s}{2}}}\|u\|_{\dot{H}^{s}}^{1-\frac{2 s}{N}} \text { with } \quad \frac{1}{r}=\frac{1}{2}-\frac{s}{N} .
$$


The following result indicates the invariance of (1.12) and (1.13) under oscillations.

Proposition 1.5. Let $\theta$ be a function in $\mathcal{S}, p$ in $[1, \infty], \sigma$ in $]-N(1-1 / p),+\infty[$ and $\varepsilon_{0}$ a positive real number. There exists a constant $C$ such that the oscillatory function $f_{\varepsilon}(w) \stackrel{\text { def }}{=} \theta(w) e^{i w_{1} / \varepsilon}$ satisfies

$$
\forall \varepsilon \leq \varepsilon_{0},\left\|f_{\varepsilon}\right\|_{\dot{B}_{p, 1}^{\sigma}} \leq C \varepsilon^{-\sigma}
$$

This proposition implies immediately the following corollary.

Corollary 1.6. There exists a family $\left(f_{\varepsilon}\right)_{\varepsilon>0}$ of smooth functions such that, for any $s$ in $] 0, N / 2$ [ and any $\beta>2 s / N$, we have

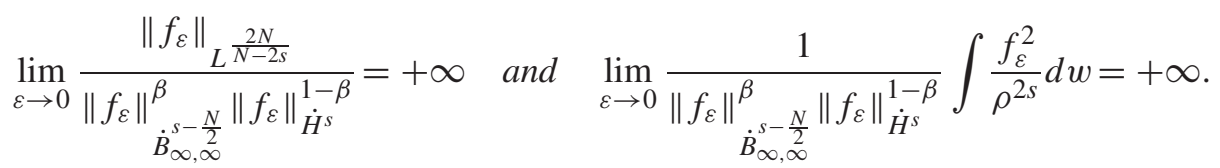

\subsection{Structure of the paper and idea of the proof}

The idea of the proof of Theorems 1.2 and 1.4 is to see them from a non linear point of view. More precisely, we write

$$
\int \frac{u^{2}(w)}{\rho^{2 s}(w)} d w=\left\langle\rho^{-2 s}, u^{2}\right\rangle .
$$

Then it is enough to prove that $\rho^{-2 s}$ and $u^{2}$ belongs to a pair of spaces in duality.

In the second section, we shall prove that $\rho^{-2 s}$ belongs to the space $\dot{B}_{1, \infty}^{N-2 s}$. Then using a product law, we shall conclude the proof of Theorem 1.2.

In the third section, we shall use paradifferential calculus to prove Theorem 1.4.

In the fourth section, we shall prove Proposition 1.5. We shall also investigate if it is possible to extend Corollary 1.6 for a family of non negative functions.

\section{The behavior of negative powers of $\rho$}

It is described by the following proposition.

Proposition 2.1. Let $s$ be a real number in the interval ]0, $N / 2[$. Then the function $\rho^{-2 s}$ belongs to the Besov space $\dot{B}_{1, \infty}^{N-2 s}$.

Proof of Proposition 2.1. Let us introduce a smooth compactly supported function $\chi$ which is identically equal to 1 near the unit ball and let us write

$$
\rho^{-2 s}=\rho_{0}+\rho_{1} \quad \text { with } \quad \rho_{0} \stackrel{\text { def }}{=} \chi \rho^{-2 s} \quad \text { and } \quad \rho_{1} \stackrel{\text { def }}{=}(1-\chi) \rho^{-2 s} \text {. }
$$


It is obvious that $\rho^{-2 s} \in L^{1}+L^{q}$ with $q>N / 2 s$ which implies that $\lim _{j \rightarrow-\infty} S_{j} \rho^{-2 s}=$ 0 in $L^{1}+L^{q}$. Then, the homogeneity of the function $\rho$ gives

$$
\begin{aligned}
\Delta_{j} \rho^{-2 s} & =2^{j N} \rho^{-2 s} \star h\left(\delta_{2^{j}} \cdot\right) \\
& =2^{j(N+2 s)} \rho^{-2 s}\left(\delta_{2^{j}} \cdot\right) \star h\left(\delta_{2^{j}} \cdot\right) \\
& =2^{2 j s}\left(\Delta_{0} \rho^{-2 s}\right)\left(\delta_{2^{j}} \cdot\right) .
\end{aligned}
$$

Therefore $\left\|\Delta_{j} \rho^{-2 s}\right\|_{L^{1}}=2^{j(2 s-N)}\left\|\Delta_{0} \rho^{-2 s}\right\|_{L^{1}}$ which reduces the problem to proving that the function $\Delta_{0} \rho^{-2 s}$ is in $L^{1}$. As $\rho_{0}$ is in $L^{1}, \Delta_{0} \rho_{0}$ is also in $L^{1}$ thanks to the continuity of the operator $\Delta_{0}$ on Lebesgue spaces. In order to estimate $\rho_{1}$ in $L^{1}$, we shall use Inequality (1.9) to write that

$$
\left\|\Delta_{0} \rho_{1}\right\|_{L^{1}} \leq C_{k}\left\|(-\Delta)^{k} \Delta_{0} \rho_{1}\right\|_{L^{1}} \leq C_{k}\left\|(-\Delta)^{k} \rho_{1}\right\|_{L^{1}} .
$$

By the Leibniz formula, $(-\Delta)^{k} \rho_{1}-(1-\chi)(-\Delta)^{k} \rho$ is a smooth compactly supported function. Then, we achieve the proof by using (1.4) and choosing $k$ such that $2 k>N-2 s$.

As an application, we shall prove Theorem 1.2. When $u$ belongs to $\dot{H}^{s}$, then

$$
u^{2} \in \dot{B}_{2,1}^{2 s-\frac{N}{2}} \quad \text { and } \quad\left\|u^{2}\right\|_{\dot{B}_{2,1}^{2 s-\frac{N}{2}}} \leq C\|u\|_{\dot{H}^{s}}^{2} .
$$

That result is classical in $\mathbf{R}^{N}$ and was proved in $\mathbf{H}^{d}$ by two of the authors in [3]. Now writing that

$$
\left\langle\rho^{-2 s}, u^{2}\right\rangle=\sum_{\left|j-j^{\prime}\right| \leq N_{0}}\left\langle\Delta_{j} \rho^{-2 s}, \Delta_{j^{\prime}} u^{2}\right\rangle,
$$

we infer, thanks to Proposition 2.1 and embeddings (1.11), that

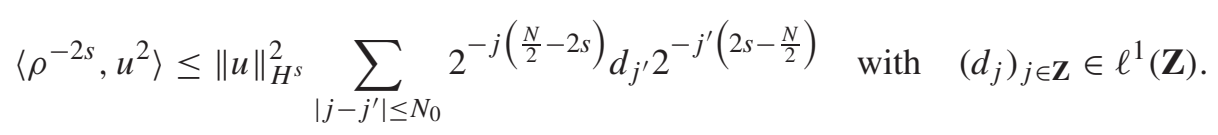

This proves Theorem 1.2.

Remark 2.2. Let us point out that, in Theorem 1.2, the function $\rho^{-2 s}$ can be any function in $\dot{B}_{2, \infty}^{\frac{N}{2}-2 s}$.

\section{Paradifferential calculus and refined inequalities}

In order to prove Theorem 1.4, let us recall the paraproduct algorithm introduced by J.-M. Bony in [4] in the case of $\mathbf{R}^{N}$ and by two of the authors in the case of $\mathbf{H}^{d}$ 
in [3]. In both cases, this allows to write that

$$
\begin{aligned}
& u^{2}=2 T_{u} u+R(u, u), \quad \text { with } \quad T_{u} u \stackrel{\text { def }}{=} \sum_{j} S_{j-N_{0}} u \Delta_{j} u \\
& \text { and } \quad R(u, u) \stackrel{\text { def }}{=} \sum_{\left|j-j^{\prime}\right| \leq N_{0}} \Delta_{j} u \Delta_{j^{\prime}} u .
\end{aligned}
$$

Using (1.7) and (1.6), we get

$$
\begin{aligned}
\left\|\Delta_{j} T_{u} u\right\|_{L^{\infty}} & =\left\|\sum_{\left|j-j^{\prime}\right| \leq N_{0}} \Delta_{j}\left(S_{j^{\prime}-N_{0}} u \Delta_{j^{\prime}} u\right)\right\|_{L^{\infty}} \\
& \leq \sum_{\left|j-j^{\prime}\right| \leq N_{0}}\left\|S_{j^{\prime}-N_{0}} u\right\|_{L^{\infty}\left\|\Delta_{j^{\prime}} u\right\|_{L^{\infty}} .}
\end{aligned}
$$

Now let us write that

$$
2^{-j\left(\frac{N}{2}-s\right)}\left\|S_{j} u\right\|_{L^{\infty}} \leq \sum_{k \leq j-1} 2^{(j-k)\left(s-\frac{N}{2}\right)} 2^{k\left(s-\frac{N}{2}\right)}\left\|\Delta_{k} u\right\|_{L^{\infty}} .
$$

Young's inequality on series implies that

$$
\left\|S_{j} u\right\|_{L^{\infty}} \leq C c_{j} 2^{j\left(\frac{N}{2}-s\right)}\|u\|_{\dot{B}_{\infty, 2}^{s-\frac{N}{2}}} \text { with } \quad \sum_{j} c_{j}^{2}=1 .
$$

This gives

$$
\begin{aligned}
\left\|\Delta_{j}\left(T_{u} u\right)\right\|_{L^{\infty}} & \leq C\|u\|_{\dot{B}_{\infty, 2}^{s-\frac{N}{2}}} \sum_{j^{\prime}=j-N_{0}}^{j+N_{0}} 2^{j^{\prime}(N-2 s)} c_{j^{\prime}} 2^{-j^{\prime}\left(\frac{N}{2}-s\right)}\left\|\Delta_{j^{\prime}} u\right\|_{L^{\infty}} \\
& \leq C\|u\|_{\dot{B}_{\infty, 2}^{s-\frac{N}{2}}}^{2} 2^{j(N-2 s)} \sum_{j^{\prime}=j-N_{0}}^{j+N_{0}} d_{j^{\prime}} \text { with } \sum_{j^{\prime}} d_{j^{\prime}}=1 .
\end{aligned}
$$

Thanks to (1.11), and Proposition 2.1, we have,

$$
\left\langle\rho^{-2 s}, T_{u} u\right\rangle \leq C\|u\|_{\dot{B}_{q, 2}^{s-N}\left(\frac{1}{2}-\frac{1}{q}\right)}^{2 \alpha}\|u\|_{\dot{B}_{p, 2}^{s-N\left(\frac{1}{2}-\frac{1}{p}\right)}}^{2-2 \alpha}
$$

for any $0 \leq \alpha \leq 1$ and $p, q \geq 1$.

The estimate of $\left\langle\rho^{-2 s}, R(u, u)\right\rangle$ relies on the following elementary interpolation lemma. 
Proposition 3.1. Let $s$ be a real number in the interval ]0, $N / 2[$ and let $p$ and $q$ be two real numbers in $[1, \infty]$ such that

$$
2 \leq q<\frac{2 N}{N-2 s}<p \leq \infty .
$$

There is a constant $C$ such that for any functions $f$ and $g$ which belongs to $L^{p} \cap L^{q}$, we have

$$
\left\langle\rho^{-2 s}, f g\right\rangle \leq C\|f\|_{L^{p}}^{\alpha}\|g\|_{L^{p}}^{\alpha}\|f\|_{L^{q}}^{1-\alpha}\|g\|_{L^{q}}^{1-\alpha} \quad \text { with } \quad \alpha=\frac{p q}{p-q}\left(\frac{1}{q}-\frac{1}{2}+\frac{s}{N}\right) .
$$

Proof of Proposition 3.1. Let us write that, for any positive $R$,

$$
\begin{array}{r}
\left\langle\rho^{-2 s}, f g\right\rangle=I_{1}(R)+I_{2}(R) \quad \text { with } I_{1}(R) \stackrel{\text { def }}{=} \int_{(\rho \leq R)} \frac{f g}{\rho^{2 s}} d w \\
\text { and } I_{2}(R) \stackrel{\text { def }}{=} \int_{(\rho \geq R)} \frac{f g}{\rho^{2 s}} d w .
\end{array}
$$

The condition on $p$ and $q$ implies that $\rho^{-2 s}$ is locally $L^{\frac{p}{p-2}}$ and is $L^{\frac{q}{q-2}}$ outside any compact neighbourhood of 0 . By Hölder's inequality, we infer that

$$
\begin{aligned}
& I_{1}(R) \leq\left\|\mathbf{1}_{(\rho \leq R)} \rho^{-2 s}\right\|_{L^{\frac{p}{p-2}}}\|f\|_{L^{p}}\|g\|_{L^{p}} \quad \text { and } \\
& I_{2}(R) \leq\left\|\mathbf{1}_{(\rho \geq R)} \rho^{-2 s}\right\|_{L^{\frac{q}{q-2}}}\|f\|_{L^{q}}\|g\|_{L^{q}} .
\end{aligned}
$$

As the function $\rho$ is homogeneous of order 1 , we get, by the change of variable $w^{\prime}=$ $\delta_{R^{-1}} w$,

$$
\begin{aligned}
& \left\|\mathbf{1}_{(\rho \leq R)} \rho^{-2 s}\right\|_{L^{\frac{p}{p-2}}}=R^{N-2 s-\frac{2 N}{p}}\left\|\mathbf{1}_{(\rho \leq 1)} \rho^{-2 s}\right\|_{L^{\frac{p}{p-2}}} \text { and } \\
& \left\|\mathbf{1}_{(\rho \geq R)} \rho^{-2 s}\right\|_{L^{\frac{q}{q-2}}}=R^{N-2 s-\frac{2 N}{q}}\left\|\mathbf{1}_{(\rho \geq 1)} \rho^{-2 s}\right\|_{L^{\frac{q}{q-2}}} .
\end{aligned}
$$

Thus we have, for any positive $R$,

$$
\left\langle\rho^{-2 s}, f g\right\rangle \leq C R^{N-2 s}\left(R^{-\frac{2 N}{p}}\|f\|_{L^{p}}\|g\|_{L^{p}}+R^{-\frac{2 N}{q}}\|f\|_{L^{q}}\|g\|_{L^{q}}\right) .
$$

Choosing the optimal

$$
R=\left(\frac{\|f\|_{L^{q}}\|g\|_{L^{q}}}{\|f\|_{L^{p}}\|g\|_{L^{p}}}\right)^{\frac{p q}{2 N(p-q)}}
$$

concludes the proof of the proposition. 
Let us go back to the proof of Theorem 1.4. By definition of $R(u, u)$, we have

$$
\left\langle\rho^{-2 s}, R(u, u)\right\rangle=\sum_{|\ell| \leq N_{0}} \sum_{j \in \mathbf{Z}}\left\langle\rho^{-2 s}, \Delta_{j} u \Delta_{j-\ell} u\right\rangle .
$$

Proposition 3.1 implies that

$$
\begin{aligned}
\left\langle\rho^{-2 s}, R(u, u)\right\rangle \leq & \sum_{|\ell| \leq N_{0}} \sum_{j \in \mathbf{Z}}\left(2^{2 j\left(s-N\left(\frac{1}{2}-\frac{1}{p}\right)\right)}\left\|\Delta_{j} u\right\|_{L^{p}}\left\|\Delta_{j-\ell} u\right\|_{L^{p}}\right)^{\alpha} \\
& \times\left(2^{2 j\left(s-N\left(\frac{1}{2}-\frac{1}{q}\right)\right)}\left\|\Delta_{j} u\right\|_{L^{q}}\left\|\Delta_{j-\ell} u\right\|_{L^{q}}\right)^{1-\alpha}
\end{aligned}
$$

By definition of the Besov norms, this implies that two series $\left(c_{j}\right)_{j \in \mathbf{Z}}$ and $\left(c_{j}^{\prime}\right)_{j \in \mathbf{Z}}$ exist in the unit sphere of $\ell^{2}(\mathbf{Z})$ such that

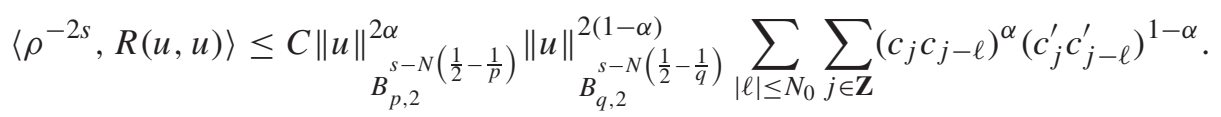

From Hölder inequalities, it follows that

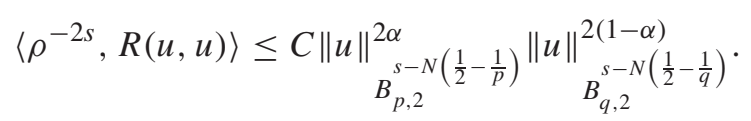

Together with (3.1), this gives Theorem 1.4.

\section{Oscillations and fractal transforms in refined inequalities}

The purpose of this section is to provide examples which show that the refined estimates are sharp. The first one deals with oscillating functions.

\subsection{Oscillations}

Here we want to prove Proposition 1.5, namely, by definition of Besov spaces, that for any function $\theta$ in $\mathcal{S}$, we have

$$
\sum_{j} 2^{j \sigma}\left\|\Delta_{j} f_{\varepsilon}\right\|_{L^{p}} \leq C \varepsilon^{-\sigma} \quad \text { with } \quad f_{\varepsilon}(w) \stackrel{\text { def }}{=} e^{i \frac{w_{1}}{\varepsilon}} \theta(w)
$$

We shall treat differently the high frequencies (indices $j$ such that $2^{j} \varepsilon$ is greater than 1) and low frequencies (indices $j$ such that $2^{j} \varepsilon$ is less than 1).

Let us first estimate the low frequencies. Denoting the vector $\widetilde{Z}_{1}=\partial_{x_{1}}$ in the case of $\mathbf{R}^{N}$ and $\widetilde{Z}_{1}=\partial_{x_{1}}-y_{1} \partial_{s}$ in the case of $\mathbf{H}^{d}$, we have

$$
i \varepsilon \widetilde{Z}_{1} e^{i \frac{w_{1}}{\varepsilon}}=-e^{i \frac{w_{1}}{\varepsilon}}
$$


By integration by parts, we get

$$
\begin{aligned}
\Delta_{j} f_{\varepsilon}\left(w^{\prime}\right) & =(-i \varepsilon)^{N} 2^{j N} \int\left(-\widetilde{Z}_{1}\right)^{N}\left(e^{i \frac{w_{1}^{\prime}-w_{1}}{\varepsilon}}\right) \theta\left(w^{\prime} w^{-1}\right) h\left(\delta_{2^{j}}(w)\right) d w \\
& =(-i \varepsilon)^{N} 2^{j N} \int e^{i \frac{w_{1}^{\prime}-w_{1}}{\varepsilon}}\left(\widetilde{Z}_{1}\right)^{N}\left(\theta\left(w^{\prime} w^{-1}\right) h\left(\delta_{2^{j}}(w)\right) d w\right. \\
& =(-i \varepsilon)^{N} 2^{j N} \sum_{\ell=0}^{N} C_{N}^{\ell} \int e^{i \frac{w_{1}^{\prime}-w_{1}}{\varepsilon}} \widetilde{Z}_{1}^{N-\ell}\left(\theta\left(w^{\prime} w^{-1}\right)\right) \widetilde{Z}_{1}^{\ell}\left(h\left(\delta_{2^{j}}(w)\right) d w\right.
\end{aligned}
$$

where the vector field $\widetilde{Z}_{1}$ acts on the variable $w$. As

$$
\widetilde{Z}_{1}\left(h\left(\delta_{2^{j}}(w)\right)=2^{j}\left(\widetilde{Z}_{1} h\right)\left(\delta_{2^{j}}(w)\right) \text { and } \quad-\widetilde{Z}_{1}\left(\theta\left(w^{\prime} w^{-1}\right)\right)=\left(Z_{1} \theta\right)\left(w^{\prime} w^{-1}\right),\right.
$$

we infer that

$$
\begin{aligned}
& \left|\Delta_{j} f_{\varepsilon}\left(w^{\prime}\right)\right| \\
= & \varepsilon^{N} 2^{j N}\left|\sum_{\ell=0}^{N} C_{N}^{\ell} 2^{j \ell}(-1)^{N-\ell} \int e^{i \frac{w_{1}^{\prime}-w_{1}}{\varepsilon}}\left(Z_{1}^{N-\ell} \theta\right)\left(w^{\prime} w^{-1}\right)\left(\widetilde{Z}_{1}^{\ell} h\right)\left(\delta_{2^{j}}(w)\right) d w\right| \\
\leq & \varepsilon^{N} 2^{j N} \sum_{\ell=0}^{N} C_{N}^{\ell} 2^{j \ell}\left(\left|Z_{1}^{N-\ell} \theta\right| \star\left|\left(\widetilde{Z}_{1}^{\ell} h\right)\left(\delta_{2^{j}} \cdot\right)\right|\right)\left(w^{\prime}\right) .
\end{aligned}
$$

Young inequalities imply that

$$
\begin{aligned}
& \left\|\left|Z_{1}^{N-\ell} \theta\right| \star\left|\left(\widetilde{Z}_{1}^{\ell} h\right)\left(\delta_{2^{j} \cdot}\right)\right|\right\|_{L^{p}} \\
& \leq \min \left\{2^{-j \frac{N}{p}}\left\|Z_{1}^{N-\ell} \theta\right\|_{L^{1}}\left\|\widetilde{Z}_{1}^{\ell} h\right\|_{L^{p}}, 2^{-j N}\left\|Z_{1}^{N-\ell} \theta\right\|_{L^{p}}\left\|\widetilde{Z}_{1}^{\ell} h\right\|_{L^{1}}\right\} .
\end{aligned}
$$

Therefore, as $\sigma>-N\left(1-\frac{1}{p}\right)$,

$$
\begin{aligned}
\sum_{2^{j} \leq \frac{1}{\varepsilon}} 2^{j \sigma}\left\|\Delta_{j} f_{\varepsilon}\right\|_{L^{p}} & \leq C \varepsilon^{N}\left(\sum_{2^{j} \leq 1} 2^{j\left(\sigma+N\left(1-\frac{1}{p}\right)\right)}+\sum_{1 \leq 2^{j} \leq \frac{1}{\varepsilon}} 2^{j(\sigma+N)}\right) \\
& \leq C \varepsilon^{-\sigma}
\end{aligned}
$$

In order to estimate high frequencies, let us use (1.9). We get, for any non negative integer $M$,

$$
\begin{aligned}
\left\|\Delta_{j} f_{\varepsilon}\right\|_{L^{p}} & \leq C 2^{j(N-2 M)}\left\|\left((-\Delta)^{M} f_{\varepsilon}\right) \star h\left(\delta_{2^{j}} \cdot\right)\right\|_{L^{p}} \\
& \leq C 2^{-2 j M}\left\|(-\Delta)^{M} f_{\varepsilon}\right\|_{L^{p}} .
\end{aligned}
$$


The Leibniz formula implies that, for any $\left.\varepsilon \in] 0, \varepsilon_{0}\right],\left\|(-\Delta)^{M} f_{\varepsilon}\right\|_{L^{p}} \leq C \varepsilon^{-2 M}\|\theta\|_{L^{p}}$. Thus we infer, thanks to (1.6), that

$$
\sum_{2^{j} \geq \frac{1}{\varepsilon}} 2^{j \sigma}\left\|\Delta_{j} f_{\varepsilon}\right\|_{L^{p}} \leq C \varepsilon^{-2 M} \sum_{2^{j} \geq \frac{1}{\varepsilon}} 2^{j(\sigma-2 M)} .
$$

Choosing $M$ such that $\sigma-2 M$ is negative gives

$$
\sum_{2^{j} \geq \frac{1}{\varepsilon}} 2^{j \sigma}\left\|\Delta_{j} f_{\varepsilon}\right\|_{L^{p}} \leq C \varepsilon^{-\sigma}
$$

This ends the proof of Proposition 1.5.

\subsection{Fractal transform and Besov norms}

In this subsection we will show that oscillations are not the sole responsible for the smallness of a Besov norm. Below we present another situation, of a sequence of non negative functions for which the $L^{p}$ norms and the Besov norms are balanced as the family $\left(f_{\varepsilon}\right)$ of Proposition 1.5. Again, we shall present statements and proofs common to the case of $\mathbf{R}^{N}$ and $\mathbf{H}^{d}$. In order to do so, let us define the distance $d$ as

$$
\forall\left(w, w^{\prime}\right) \in \mathbf{R}^{N} \times \mathbf{R}^{N}, \quad d\left(w, w^{\prime}\right) \stackrel{\text { def }}{=} \max _{1 \leq j \leq N}\left|w_{j}-w_{j}^{\prime}\right|
$$

and, for any $\left(w, w^{\prime}\right) \in \mathbf{H}^{d} \times \mathbf{H}^{d}$,

$d\left(w, w^{\prime}\right) \stackrel{\text { def }}{=} \max \left\{\max _{1 \leq j \leq d}\left|x_{j}-x_{j}^{\prime}\right|, \max _{1 \leq j \leq N}\left|y_{j}-y_{j}^{\prime}\right|,\left|s-s^{\prime}+\left(y^{\prime} \mid x\right)-\left(y \mid x^{\prime}\right)\right|^{\frac{1}{2}}\right\}$,

where in the case of $\mathbf{H}^{d}$ we have noted $w=(x, y, s)$ and $w^{\prime}=\left(x^{\prime}, y^{\prime}, s^{\prime}\right)$. Let us denote by $Q$ the ball for $d$ centered at zero and of radius $1 / 2$. Now let us define the following quantities. Let $D$ and $L$ such that $D=L=N$ in the case of $\mathbf{R}^{N}$ and $D=N-1$ and $L=N+1$ in the case of $\mathbf{H}^{d}$. For $J$ in $\{-1,1\}^{L}$, we define the point $w_{J}$ of $Q$ and the cube $Q_{J}$ by

$$
w_{J} \stackrel{\text { def }}{=} \delta_{\frac{3}{8}} J \quad \text { and } \quad Q_{J} \stackrel{\text { def }}{=} w_{J} \cdot \delta_{\frac{1}{4}} Q=\left\{w / d\left(w, w_{J}\right) \leq \frac{1}{8}\right\} .
$$

Omitted elementary computations show that

$$
Q_{J} \subset Q \quad \text { and } \quad\left(J \neq J^{\prime} \Longrightarrow d\left(Q_{J}, Q_{J^{\prime}}\right) \geq \frac{\sqrt{3}}{4}\right)
$$

Now let us define the transform $T$ which duplicates (after dilation and translation) functions defined on $Q$. 
Definition 4.1. Let us denote by $T$ the following transform

$$
T\left\{\begin{aligned}
\mathcal{D}(Q) & \rightarrow \mathcal{D}(Q) \\
f & \mapsto T f \stackrel{\text { def }}{=} 2^{D} \sum_{J \in\{-1,1\}^{L}} f_{J} \quad \text { with } \quad f_{J}(w) \stackrel{\text { def }}{=} f\left(\delta_{4}\left(w_{J}^{-1} w\right)\right) .
\end{aligned}\right.
$$

For a subset $A$ of $Q$, we denote by $T A$ the set defined by

$$
T A \stackrel{\text { def }}{=} \bigcup_{J \in\{-1,1\}^{L}} w_{J} \delta_{\frac{1}{4}} A .
$$

Let us notice that $T A \subset Q$ and that $\operatorname{Supp}(T f)=T$ (Supp $f$ ). Let us also observe that, using (4.3), we have

$$
\begin{aligned}
\|T f\|_{L^{p}}^{p} & =2^{D p} \sum_{J \in\{-1,1\}^{L}}\left\|f_{J}\right\|_{L^{p}}^{p} \\
& =2^{D p}\left(\sum_{J \in\{-1,1\}^{L}} 2^{-2 N}\right)\|f\|_{L^{p}}^{p} \\
& =2^{D p+L-2 N}\|f\|_{L^{p}}^{p} \\
& =2^{D(p-1)}\|f\|_{L^{p}}^{p} .
\end{aligned}
$$

Thus, we have

$$
\|T f\|_{L^{p}}=2^{D\left(1-\frac{1}{p}\right)}\|f\|_{L^{p}} .
$$

The way $T$ acts on Besov spaces is described by the following proposition.

Proposition 4.2. For any $(p, r) \in[1,+\infty]^{2}$ and any $\sigma$ in $]-N\left(1-\frac{1}{p}\right),+\infty[$, there exists a constant $C$ such that

$$
\|T f\|_{\dot{B}_{p, r}^{\sigma}} \leq 2^{D\left(1-\frac{1}{p}\right)+2 \sigma}\|f\|_{\dot{B}_{p, r}^{\sigma}}+C\|f\|_{L^{1}} .
$$

Proof of Proposition 4.2. For the sake of simplicity, we only prove this proposition in the case when $r=1$. By definition of the Besov norm, we have

$$
\begin{array}{r}
\|T f\|_{\dot{B}_{p, 1}^{\sigma}}=T_{1} f+T_{2} f \quad \text { with } \quad T_{1} f \stackrel{\text { def }}{=} \sum_{j \leq 0} 2^{j \sigma}\left\|\Delta_{j} T f\right\|_{L^{p}} \\
\text { and } T_{2} f \stackrel{\text { def }}{=} \sum_{j \geq 1} 2^{j \sigma}\left\|\Delta_{j} T f\right\|_{L^{p}} .
\end{array}
$$

On the one hand, using Bernstein's inequality (1.6), the fact that $N\left(1-\frac{1}{p}\right)+\sigma>0$ and (4.4) with $p=1$, we get

$$
T_{1} f \leq C \sum_{j \leq 0} 2^{j \sigma+j N\left(1-\frac{1}{p}\right)}\left\|\Delta_{j} T f\right\|_{L^{1}} \leq C\|T f\|_{L^{1}} \leq C\|f\|_{L^{1}}
$$


The estimate on $T_{2} f$ uses the special structure of $T Q$. Let us define the set $\widetilde{Q} \stackrel{\text { def }}{=}\left\{w / d(w, T Q) \leq \frac{1}{32}\right\}=\bigcup_{J \in\{-1,1\}^{L}} \widetilde{Q}_{J} \quad$ with $\quad \widetilde{Q}_{J} \stackrel{\text { def }}{=}\left\{w / d\left(w, Q_{J}\right) \leq \frac{1}{32}\right\}$

Now let us write that $T_{2} f=T_{21} f+T_{22} f$ with

$$
T_{21} f \stackrel{\text { def }}{=} \sum_{j \geq 1} 2^{j \sigma}\left\|\Delta_{j} T f\right\|_{L^{p}\left({ }^{c} \widetilde{Q}\right)} \quad \text { and } \quad T_{22} f \stackrel{\text { def }}{=} \sum_{j \geq 1} 2^{j \sigma}\left\|\Delta_{j} T f\right\|_{L^{p}(\tilde{Q})} .
$$

Let us recall that

$$
\left(\Delta_{j} T f\right)\left(w^{\prime}\right)=2^{j N} \int T f(w) h\left(\delta_{2^{j}}\left(w^{-1} w^{\prime}\right)\right) d w .
$$

As $h$ belongs to $\mathcal{S}$, we have, for any positive integer $M$, that $|h(w)| \leq C_{M}(1+$ $\rho(w))^{-M}$. Thus, by homogeneity and by definition of $\rho$ and $d$, we get, for all $\left(w, w^{\prime}\right) \in T Q \times \widetilde{Q}^{c}$,

$$
\begin{aligned}
\left|h\left(\delta_{2^{j}}\left(w^{-1} w^{\prime}\right)\right)\right| & \leq C_{M}\left(1+\rho\left(\delta_{2^{j}}\left(w^{-1} w^{\prime}\right)\right)\right)^{-N-1} \rho^{-M}\left(\delta_{2^{j}}\left(w^{-1} w^{\prime}\right)\right) \\
& \leq C_{M}\left(1+\rho\left(\delta_{2^{j}}\left(w^{-1} w^{\prime}\right)\right)\right)^{-N-1} 2^{-j M} \rho^{-M}\left(w^{-1} w^{\prime}\right) \\
& \leq C_{M} 2^{-j M}\left(1+\rho\left(\delta_{2^{j}}\left(w^{-1} w^{\prime}\right)\right)\right)^{-N-1} .
\end{aligned}
$$

Using (4.4), we infer that, for any integer $M$,

$$
\begin{aligned}
\left\|\Delta_{j} T f\right\|_{L^{p}\left({ }^{c} \widetilde{Q}\right)} & \leq C_{M} 2^{j\left(N\left(1-\frac{1}{p}\right)-M\right)}\|T f\|_{L^{1}} \\
& \leq C_{M} 2^{j\left(N\left(1-\frac{1}{p}\right)-M\right)}\|f\|_{L^{1}} .
\end{aligned}
$$

Then, choosing $M$ large enough, we infer

$$
T_{21} f \leq C\|f\|_{L^{1}}
$$

Finally let us estimate $T_{22} f$. As $\widetilde{Q}$ is the disjoint union of the $\widetilde{Q}_{J}$, we get

$$
\left\|\Delta_{j} T f\right\|_{L^{p}(\widetilde{Q})} \leq 2^{\frac{L}{p}} \sup _{J \in\{-1,1\}^{L}}\left\|\Delta_{j} T f\right\|_{L^{p}\left(\widetilde{Q}_{J}\right)}
$$

Let us first estimate $\left\|\Delta_{j} f_{J^{\prime}}\right\|_{L^{p}\left(\widetilde{Q}_{J}\right)}$ for $J^{\prime} \neq J$. We have, for all $w^{\prime} \in \widetilde{Q}_{J}$,

$$
\left(\Delta_{j} f_{J^{\prime}}\right)\left(w^{\prime}\right)=2^{j N} \int f_{J^{\prime}}(w) h\left(\delta_{2^{j}}\left(w^{-1} w^{\prime}\right)\right) d w
$$


and in the integral, the distance $d\left(w, w^{\prime}\right)$ is greater than $1 / 32$. Then, reasoning as above we find that, for any positive integers $M$, there exists a constant $C_{M}$ such that

$$
J \neq J^{\prime} \Longrightarrow\left\|\Delta_{j} f_{J^{\prime}}\right\|_{L^{p}\left(\widetilde{Q}_{J}\right)} \leq C_{M} 2^{j\left(N\left(1-\frac{1}{p}\right)-M\right)}\left\|f_{J^{\prime}}\right\|_{L^{1}}
$$

Then, let us observe that $\left\|\Delta_{j} f_{J}\right\|_{L^{p}\left(\widetilde{Q}_{J}\right)} \leq\left\|\Delta_{j} f_{J}\right\|_{L^{p}}$. Writing that

$$
w^{-1} w^{\prime}=\delta_{\frac{1}{4}}\left(\left(\delta_{4}\left(w_{J}^{-1} w\right)\right)^{-1} \delta_{4}\left(w_{J}^{-1} w^{\prime}\right)\right)
$$

and changing variable $v=\delta_{4}\left(w_{J}^{-1} w\right)$ gives

$$
\left(\Delta_{j} f_{J}\right)\left(w^{\prime}\right)=\left(\Delta_{j-2} f\right)\left(\delta_{4}\left(w_{J}^{-1} w^{\prime}\right)\right) .
$$

Thus $\left\|\Delta_{j} f_{J}\right\|_{L^{p}}=2^{-\frac{2 N}{p}}\left\|\Delta_{j-2} f\right\|_{L^{p}}$. Then using (4.7) and (4.8), we get by definition of $T$, that for all positive integers $M$,

$$
\left\|\Delta_{j} f\right\|_{L^{p}(\widetilde{Q})} \leq 2^{D+\frac{L-2 N}{p}}\left\|\Delta_{j-2} f\right\|_{L^{p}}+C_{M} 2^{j\left(N\left(1-\frac{1}{p}\right)-M\right)}\|f\|_{L^{1}} .
$$

Thus by definition of $T_{22} f$, we get by choosing $M$ large enough,

$$
\begin{aligned}
T_{22} f & \leq 2^{D\left(1-\frac{1}{p}\right)} \sum_{j \geq 1} 2^{j \sigma}\left\|\Delta_{j-2} f\right\|_{L^{p}}+C_{M}\|f\|_{L^{1}} \sum_{j \geq 1} 2^{j\left(\sigma+N\left(1-\frac{1}{p}\right)-M\right)} \\
& \leq 2^{D\left(1-\frac{1}{p}\right)+2 \sigma}\|f\|_{\dot{B}_{p, 1}^{\sigma}}+C\|f\|_{L^{1}} .
\end{aligned}
$$

Together with (4.5) and (4.6), this concludes the proof of the proposition.

Let us state the following corollary of Proposition 4.2.

Corollary 4.3. For $(N-D) / 2<s<N / 2$, there exists a sequence $\left(f_{n}\right)_{n \in \mathbf{N}}$ of non negative smooth and compactly supported functions such that, for any $\beta>2 s / N$,

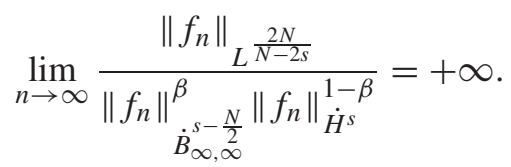

Proof of Corollary 4.3. Let us consider a smooth compactly supported non negative function $f_{0}$ and let us define the sequence $\left(f_{n}\right)_{n \in \mathbf{N}}$ by $f_{n}=T^{n} f_{0}$. By iteration of the inequality of Proposition 4.2, we have

$$
\left.\left\|f_{n}\right\|_{B_{p, q}^{\sigma}} \leq 2^{n\left(D\left(1-\frac{1}{p}\right)+2 \sigma\right.}\right)\left\|f_{0}\right\|_{B_{p, q}^{\sigma}}+C\left(\sum_{m=0}^{n-1} 2^{m\left(D\left(1-\frac{1}{p}\right)+2 \sigma\right)}\right)\left\|f_{0}\right\|_{L^{1}} .
$$


If $\sigma>-\frac{D}{2}\left(1-\frac{1}{p}\right)$, we deduce that

$$
\left\|f_{n}\right\|_{B_{p, q}^{\sigma}} \leq C_{f_{0}} 2^{n\left(D\left(1-\frac{1}{p}\right)+2 \sigma\right)}
$$

Applying this first with $\sigma=s-N / 2$ and $p=q=\infty$ and then with $\sigma=s$ and $p=q=2$ gives

$$
\left\|f_{n}\right\|_{\dot{B}_{\infty, \infty}^{s-\frac{N}{2}}} \leq C_{f_{0}} 2^{n(D-N+2 s)} \quad \text { and } \quad\left\|f_{n}\right\|_{\dot{H}^{s}} \leq C_{f_{0}} 2^{n\left(\frac{D}{2}+2 s\right)} .
$$

Assertion (4.4) claims that

$$
\left\|f_{n}\right\|_{L^{\frac{2 N}{N-2 s}}}=2^{n D\left(\left(\frac{1}{2}+\frac{s}{N}\right)\right)}\left\|f_{0}\right\|_{L^{\frac{2 N}{N-2 s}}} .
$$

This concludes the proof of the corollary.

Remark 4.4. Unfortunately, we cannot claim the same result for the refined Hardy inequality. Let us notice that the refined Hardy inequality has an obvious translation invariant generalization which is

$$
\sup _{a} \int \frac{u^{2}(w)}{\rho^{2 s}\left(a^{-1} w\right)} d w \leq C\|u\|_{\dot{B}_{\infty, 2}^{s-\frac{N}{2}}}^{\frac{2 s}{N}}\|u\|_{\dot{H}^{s}}^{1-\frac{2 s}{N}} .
$$

For the sequences used in the proof of Corollary 4.3, omitted computations show that, if $s$ is greater than $\frac{1}{2}\left(N-\frac{D}{2}\right)$,

$$
\sup _{a} \int \frac{f_{n}^{2}(w)}{\rho^{2 s}\left(a^{-1} w\right)} d w \leq C 2^{n(D-N+2 s)} .
$$

This is exactly the same behavior as $\left\|f_{n}\right\|_{\dot{B}_{\infty, 2}^{s-\frac{N}{2}}}$. We do not know if the exponent can be improved in (1.12) when we restrict to the cone of non negative functions.

\section{References}

[1] H. BAhouri, J.-Y. Chemin and C.-J. XU, Trace and trace lifting theorems in weighted Sobolev spaces, J. Inst. Math. Jussieu 4 (2005), 509-552.

[2] H. BAhouri, P. GÉRARD and C.-J. XU, Espaces de Besov et estimations de Strichartz. généralisées sur le groupe de Heisenberg, J. Anal. Math. 82 (2000), 93-118.

[3] H. BAHOURI and I. Gallagher, Paraproduit sur le groupe de Heisenberg et applications, Rev. Mat. Iberoamericana 17 (2001), 69-105.

[4] J.-M. Bony, Calcul symbolique et propagation des singularités pour les équations aux dérivées partielles non linéaires, Ann. Sci. École Norm. Sup. 14 (1981), 209-246. 
[5] C. E. CAnCelier, J.-Y. Chemin and C.-J. Xu, Calcul de Weyl-Hörmander et opérateurs sous-elliptiques, Ann. Inst. Fourier (Grenoble) 43 (1993), 1157-1178.

[6] J.-Y. CHEMIN, "Fluides Parfaits Incompressibles", Astérisque, Vol. 230, 1995.

[7] J.-Y. Chemin and C.-J. XU, Inclusions de Sobolev en calcul de Weyl-Hörmander et champs de vecteurs sous-elliptiques, Ann. Sci. École Norm. Sup. 30 (1997), 719-751.

[8] J. Faraut and) K. Harzallah, "Deux Cours D'Analyse Harmonique", École d'Été d'analyse harmonique de Tunis, 1984. Progress in Mathematics, Birkhäuser.

[9] D. GELlER, Fourier analysis on the Heisenberg groups, Proc. Natl. Acad. Sciences U.S.A, 74 (1977), 1328-1331.

[10] P. GÉRARD, Y. MEYER and F. ORU, Inégalités de Sobolev précisées, Séminaire EDP, École Polytechnique, France, Décembre 1996.

[11] G. H. HARDY, Note on a theorem of Hilbert, Math. Zeit., 6 (1920), 314-317.

[12] G. H. HARDY, An inequality between integrals, Messenger of Maths. 54 (1925), 150-156.

[13] D. Jerison, The Poincaré inequality for vector fields satisfying Hörmander's conditions, Duke Math. J., 53 (1986), 503-523.

[14] D. JERISON, The Dirichlet problem for the Kohn Laplacian on the Heisenberg group, I, J. Funct. Anal. 43 (1981), 97-142.

[15] D. JERISON, The Dirichlet problem for the Kohn Laplacian on the Heisenberg group, II, J. Funct. Anal. 43 (1981), 224-257.

[16] A. I. Nachman, The Wave Equation on the Heisenberg Group, Comm. Partial Differential Equations 7 (1982), 675-714.

[17] L. RothSCHILD and E. STEIN, Hypoelliptic differential operators and nilpotent groups, Acta Math. 137 (1976), 247-320.

[18] E.M. STEIN, "Harmonic Analysis", Princeton University Press, 1993.

[19] M. E. TAYLOR, "Noncommutative Harmonic Analysis", Mathematical Surveys and Monographs, Vol. 22, AMS, Providence, Rhode Island, 1986.

Faculté des Sciences de Tunis

Département de Mathématiques

1060 Tunis, Tunisie

hajer.bahouri@fst.rnu.tn

Laboratoire J.-L. Lions UMR 7598

Université Paris VI

175 , rue du Chevaleret

75013 Paris, France

chemin@ann.jussieu.fr

Institut de Mathématiques de Jussieu UMR 7586

Université Paris VII

175, rue du Chevaleret

75013 Paris, France

Isabelle.Gallagher@math.jussieu.fr 